\title{
Clinical diagnosis of adult patients with acute megakaryocytic leukemia
}

\author{
GUANGJIE ZHAO ${ }^{1}$, WANLING WU ${ }^{1}$, XIAOQIN WANG ${ }^{1}$ and JINGWEN GU ${ }^{2}$ \\ ${ }^{1}$ Department of Hematology; ${ }^{2}$ Worldwide Medical Center, Huashan Hospital, Fudan University, Shanghai 200040, P.R. China
}

Received November 8, 2017; Accepted July 20, 2018

DOI: $10.3892 / \mathrm{ol} .2018 .9501$

\begin{abstract}
Acute megakaryocytic leukemia (AMKL) is a rare subtype of acute myeloid leukemia (AML), which is challenging to diagnose due to frequent myelofibrosis $(\mathrm{MF})$ and a low percentage of blast cells. In the present study, clinical characteristics and experimental observations in 9 adult patients diagnosed with AMKL, who were recruited by the Sino-U.S. Shanghai Leukemia Co-operative Group, were analyzed in order to summarize the diagnostic experience and provide recommendations on diagnosing AMKL. All the patients were diagnosed according to the 2008 World Health Organization diagnostic criteria. The mean age of the patients with AMKL was 59 years (range, 53-68 years). A total of 8 patients had different degrees of anemia, and 2 patients had $<5 \%$ marrow blasts present in the bone marrow; however, the percentage of positive cells with cluster of differentiation (CD)41 and CD61 expression was $>20 \%$, as demonstrated by flow cytometry. A total of 6 patients were positive for platelet-specific antigens, as indicated by immunocytochemistry. Furthermore, 7 patients presented with moderate or marked MF, as demonstrated by a bone marrow biopsy. Karyotypic analysis indicated that 6 patients had abnormal karyotypes. Only 1 patient exhibited the Janus kinase 2 V617F mutation. Treatment efficiency was notably poor, with a median survival time of 6.0 months (range, 1.1-24.0 months). In conclusion, the diagnosis of AMKL requires a combination of the results of bone marrow smears and bone marrow biopsy, immunophenotype or immunohistochemistry. We recommend that routine immunophenotypic analysis should include the CD41 and CD61 markers for diagnosing acute leukemia when bone marrow morphology does not indicate the diagnosis.
\end{abstract}

Correspondence to: Dr Jingwen Gu, Worldwide Medical Center, Huashan Hospital, Fudan University, 12 Urumqi Middle Road, Shanghai 200040, P.R. China

E-mail: jingwengu5288@163.com

Key words: acute megakaryocytic leukemia, diagnosis

\section{Introduction}

Acute megakaryocytic leukemia (AMKL) is a rare type of acute leukemia that was first reported in 1931 by Von Boros (1). In subsequent years, patients were rarely diagnosed with AMKL due to its low incidence and a lack of accurate diagnostic criteria. In 1978, Breton-Gorius et al (2) utilized immunoelectron microscopy analysis with platelet peroxidase (PPO) to identify megakaryocytes and increase the accuracy of diagnosing AMKL. AMKL was then added to the French-American-British classification as acute myeloid leukemia (AML) M7 in 1985, which identified the diagnostic criteria as exhibiting $>30 \%$ of blast cells of the bone marrow nucleated cells, which were demonstrated as a lineage derived from megakaryocytes by PPO staining (3). With the development and application of flow cytometry, the diagnosis of AMKL became more accurate. In 2008, the World Health Organization (WHO) produced precise criteria for diagnosing AMKL (4). In these criteria, AMKL was diagnosed by the presence of $\geq 20 \%$ blasts of the bone marrow nucleated cells, with $>50 \%$ of the blasts being megakaryoblasts in the bone marrow; or positive platelet-specific antigens by bone marrow aspirate or biopsy, including factor VIII, cycle of differentiation (CD)41, CD42 or CD61, as determined by immunocytochemistry staining or immunophenotyping.

In the clinic, although there are now accurate diagnostic criteria, the diagnosis of AMKL is challenging due to a high incidence of myelofibrosis (MF), resulting in difficulty in differentiating AMKL from acute MF (5). Clinical diagnostic experience with this type of leukemia is also limited. When the percentage of blast cells in the bone marrow is $<20 \%$, immunohistochemical staining and immunophenotypic analysis are not performed; therefore, the diagnosis of AMKL may lack accuracy when depending solely on the information provided by cellular morphology (6). As a result, a comprehensive diagnosis is important for this disease.

In the present study, the clinical characteristics, experimental tests and survival times of 9 adult patients with AMKL, who were recruited by the Sino-U.S. Shanghai Leukemia Cooperative Group between June 2003 and December 2010, were analyzed. Additionally, the diagnostic experience was summarized and diagnostic recommendations for AMKL were provided. 


\section{Patients and methods}

Patients. The Sino-U.S. Shanghai Leukemia Cooperative Group (School of Public Health, Fudan University) diagnosed 623 patients (Median age is 51 years old, and ranged from 18 to 88 years, the percentage of males were $56.5 \%$ ) with AML between June 2003 and December 2010, and conducted follow-ups to determine their survival time. Of these patients, 9 (1.4\%) were diagnosed with AMKL. These patients were diagnosed with AMKL according to the 2008 WHO classification criteria (4) as follows: i) The bone marrow aspirate exhibited a blast cell infiltrate comprising $\geq 20 \%$ of all cells, and $>50 \%$ of the blast cells were identified as megakaryoblasts; ii) the expression of CD41, CD42 and/or CD61 was positive, as demonstrated by flow cytometry with monoclonal or polyclonal platelet-specific antibodies; and iii) bone marrow aspiration was frequently accompanied by $\mathrm{MF}$, and in cases with fibrosis, a bone marrow biopsy was required, and the cell of origin was required to be identified as part of the megakaryocyte lineage. This was indicated by positive immunocytochemical staining for platelet-specific antigens, including factor VIII, CD41, CD42 and CD61. Patients were be diagnosed with AMKL if they met at least one of the diagnostic criteria. In the present study, all the patients with AMKL were administered one, two or more intravenous (IV) courses of a standard induction regimen that was comprised of $60 \mathrm{mg} / \mathrm{m}^{2}$ daunorubicin or $12 \mathrm{mg} / \mathrm{m}^{2}$ idarubicin per day for 3 consecutive days, plus $200 \mathrm{mg} / \mathrm{m}^{2}$ cytarabine that was continuously administered by IV infusion for 7 days. Following one course of a standard induction, a number of patients had no response and succumbed shortly after. Additionally, a number of patients had complete remission or partial remission and continued the regimen, receiving two or more courses of a standard regimen.

Diagnostic process. i) If the percentage of blast cells was $>20 \%$ in the bone marrow of nucleated cells, and cell morphology was demonstrated to be megakaryoblasts, as demonstrated using a bone marrow smear, the diagnosis was AMKL. For this diagnosis, the results of flow cytometry or immunocytochemical staining were required to increase the accuracy of the diagnosis. ii) If the bone marrow aspiration could not indicate a diagnosis of AMKL, detection methods of flow cytometry and immunocytochemical staining were vital, and the final diagnosis was frequently determined by positive platelet-specific antigens. iii) If the bone marrow aspiration diagnosis was not successful due to MF, a bone marrow biopsy was the primary test method, and the final diagnosis was determined by immunocytochemical staining for factor VIII, CD41, CD42 or CD61.

Cell morphology, bone marrow cellularity and cytochemical staining. Bone marrow aspirates and biopsies were obtained from the posterior iliac crest. Bone marrow smears were conducted by obtaining $0.2 \mathrm{ml}$ marrow aspirate from each patient, in order to perform cytomorphological classification and cytochemical staining, including myeloperoxidase, periodic acid-Schiff, $\alpha$ naphthol-acetate esterase, Sudan black B and non-specific esterase staining, according to the International Committee for Standardization in Hematology (7). Bone marrow cellularity of the aspirate and biopsy were examined by routine light microscopy (x100 magnification) and assessed with the following grading: Definite hypercellularity, normal cellularity, moderate hypocellularity and severe hypocellularity (8).

Flow cytometry immunophenotyping. An additional $3 \mathrm{ml}$ bone marrow aspirate was used for flow cytometry analysis. Mononuclear cells (MNCs) were isolated from those samples using lymphocyte separation medium (Ficoll ${ }^{\circledR}$-paque Plus; GE Healthcare Life Sciences, Chicago, IL, USA). The cells were suspended in PBS (GE HealthCare Life Sciences) with $3 \%$ bovine serum albumin (A8020; Beijing Solarbio Science $\&$ Technology Co., Ltd., Beijing, China) and was adjusted to $1 \times 10^{6} \mathrm{MNCs} / \mathrm{ml}$ and an aliquot of $100 \mu \mathrm{l}$ was labeled with 5 or $20 \mu \mathrm{l}$ fluorescein conjugated monoclonal antibodies. The applied monoclonal antibodies were directed against the following surface antigens: CD34 PE (550761, 1:5), CD45 PerCP (564105, 1:20), human leukocyte antigen DR related antigen (HLA-DR APC, 560896, 1:5), platelet-specific glycoproteins, CD41 FITC (555469, 1:5) and CD61 PE (555754, 1:5), B-cell antigens, CD10 PE (561002, 1:20) and CD19 FITC (560994, 1:5), T-cell antigens (CD2 PE (555327, 1: 20), CD3 APC $(555335,1: 5)$, CD4 APC-CY7 (561839, 1:20) and CD7 FITC (555360, 1:5), myeloid antigens, CD13 APC (561698, 1:20), CD14 PE-CY7 (560919, 1:20), CD15 FITC (555401, 1:5), CD33 PE (555450, 1:5), CD64 APC (561189, 1:20), CD117 PE (561682, 1:20) and myeloperoxidase (556035, 1:20, BD Biosciences). All monoclonal antibodies were obtained from BD Biosciences (San Jose, CA, USA). Subsequently, the cell suspension was incubated with these antibodies in the dark for $15 \mathrm{~min}$ at $4^{\circ} \mathrm{C}$, then washed in PBS with $5 \%$ EDTA and centrifuged $(200 \mathrm{x} \mathrm{g})$ for $5 \mathrm{~min}$ at $4^{\circ} \mathrm{C}$ three times. Following this, the cells were suspended in $500 \mu 1$ PBS for further use. The immunophenotyping was performed with the BD FACS Canton flow cytometer using FCS Express 3.0 software (De Novo Software, Glendale, CA, USA). Gating was set with CD45 and $90^{\circ}$ light-scatter parameters in order to exclude erythrocytes, platelets and subcellular debris. A total of $1 \times 10^{4}$ cells was acquired and the percentage of cells expressing the marker was calculated.

Immunohistochemical staining. The samples from the bone marrow biopsy were fixed in B5 stationary liquid (10\% formaldehyde) (mercuric chloride $6.0 \mathrm{~g}$, anhydrous sodium acetate $1.25 \mathrm{~g}$, distilled water $90 \mathrm{ml}$ and formaldehyde $10 \mathrm{ml}$ ) for $2 \mathrm{~h}$ at room temperature, followed by processing in $70 \%$ absolute ethyl alcohol, decalcification in Decal (EDTA bisodic salt) for $2.5 \mathrm{~h}$ at room temperature and ordinary processing with paraffin embedding. The paraffin-embedded samples were placed on slides (Dako ChernMate Capillary Gap slides; Dako; Agilent Technologies, Inc., Santa Clara, CA, USA) and stored in a warm incubator at $56^{\circ} \mathrm{C}$. After $2 \mathrm{~h}$, the samples were rinsed with water and washed with dimethylbenzene, and then, washed by graded concentration of absolute ethyl alcohol $(100,95,90,80$ and $70 \%)$. Then, the samples were sealed in $3 \%$ $\mathrm{H}_{2} \mathrm{O}_{2}$ for $10 \mathrm{~min}$ at room temperature and washed in distilled water, three times at room temperature. Subsequently, the samples underwent optimized antigen retrieval procedures, the $0.01 \mathrm{M}$ sodium citrate buffer ( $\mathrm{pH}$ 6.0) (C1010; Beijing Solarbio 
Science \& Technology Co.,Ltd.) was heated in microwave oven to $95^{\circ} \mathrm{C}$, and kept the samples in boiled sodium citrate buffer for $15 \mathrm{~min}$. After that, it was cooled at room temperature. Then the samples were washed in distilled water for $3 \mathrm{~min}$, two times at room temperature, and in PBS for 5 min, three times at room temperature, and were sealed with $5 \%$ bovine serum albumin (Gibco; Thermo Fisher Scientific, Inc., MA, USA) for $20 \mathrm{~min}$ at room temperature. The applied monoclonal antibodies (Dako; Agilent Technologies, Inc.) were against factor VIII (ab236284, 1:200, Abcam, Cambridge, MA, USA), CD42 (ab134087, 1:250, Abcam) and CD61 (ab7166, 1:250, Abcam), and were incubated with the samples overnight at $4{ }^{\circ} \mathrm{C}$. Subsequently, the sections were washed in Tris-buffered saline (TBS) three times for $10 \mathrm{~min}$ each at room temperature, and then incubated with biotinylated rabbit anti-goat secondary antibody (1:250; Vector Laboratories, Inc., Burlingame, CA, USA) for $60 \mathrm{~min}$ at room temperature, followed by washing with TBS three times for 10 min each. Following this, the sections were processed with avidin-biotin complex (TA-015-BB, Thermo Fisher Scientific Inc.) reagent for $30 \mathrm{~min}$ at room temperature, incubated in 3,3'diaminobenzidine solution (710008, Sera Care Inc, MA, USA) for $30 \mathrm{~min}$ in the dark and counterstained with hematoxylin (0701; Beijing Solarbio Science \& Technology Co., Ltd.). Subsequently, the sections were dehydrated in an ascending graded series of absolute ethyl alcohols (70, 80, 90, 95 and 100\%), cleared in xylene and cover-slipped with neutral balsam. These samples were observed in a light microscope (Nikon 801; x60 and x100) and the imaging Software NIS-Elements F 3.00, SP7 (Build 547) (both Nikon Corporation, Tokyo, Japan). The stain intensity was classified as,,,-++++++ . -, No staining is observed. + positive staining is observed in less than $25 \%$ cells. ++ positive staining in $25-49 \%$ cells. +++ extensive positive staining in more than $50 \%$ cells.

Gomori silver impregnation staining. The Gomori silver impregnation staining kit (G1800; Beijing Solarbio Science $\&$ Technology Co., Ltd.) were used. The stationary process, paraffin embedding and dehydration procedure were the same as described previously. The oxidizing agent Gomori was added to the samples for $5 \mathrm{~min}$ at room temperature, washed with running water for $30 \mathrm{sec}$. Subsequently, the samples were bleached by oxalic acid solution for $2 \mathrm{~min}$, then washed by running water for $2 \mathrm{~min}$, by distilled water for $1 \mathrm{~min}$, all the process was performed at room temperature. After that, Samples were stained with ammonium ferric sulfate solution (5\%) for $5 \mathrm{~min}$, washed by tap water for $1 \mathrm{~min}$, by distilled water for $2 \mathrm{~min}$. Silver ammonia solution was used to stain samples for $3 \mathrm{~min}$, then distilled water was used to wash the solution for $1 \mathrm{~min}$. Reducing agent of Gomori was added to the samples for $1 \mathrm{~min}$ and washed by running water for $10 \mathrm{~min}$ at room temperature. Subsequently, the sections were dehydrated in an ascending graded series of absolute ethyl alcohols $(70,80,90,95$ and $100 \%)$, cleared in xylene and cover-slipped with a neutral balsam.

Evaluation of $M F$. MF was graded according to the European Consensus Grading system (9) as follows: MF-0, scattered linear reticulin with no intersections; MF-1, loose network of reticulin with numerous intersections, particularly in perivascular areas; MF-2, diffuse and dense increases in reticulin with extensive intersections; and occasionally with only focal bundles of collagen and/or focal osteosclerosis MF-3, diffuse and dense increases in reticulin with extensive intersections, with coarse bundles of collagen, often associated with significant osteosclerosis.

Detection of the $t(9 ; 22)$ mutation. Blood samples were obtained from the posterior superior iliac spine by bone marrow aspiration and collected in a heparinized tube. Blood samples $(1 \mathrm{ml})$ were cultured in a culture dish containing $6 \mathrm{ml}$ RPMI-1640 (Hyclone; GE Healthcare Life Sciences, Logan, UT, USA) supplemented with $10 \%$ fetal calf serum (Gibco; Thermo Fisher Scientific, Inc.), $1 \%$ antibiotics and $100 \mu \mathrm{l}$ $10 \mathrm{mg} / \mathrm{ml}$ phytohemagglutinin for each patient. After $24 \mathrm{~h}$ at $37^{\circ} \mathrm{C}, 50 \mu \mathrm{l}$ colchicine $(0.1 \mathrm{mg} / \mathrm{ml})$ was added in culture dish and incubated at $37^{\circ} \mathrm{C}$ for $60 \mathrm{~min}$. Subsequently, the samples were centrifuged at $200 \mathrm{x} \mathrm{g}$ for $10 \mathrm{~min}$ at room temperature, the supernatant was removed and then $5 \mathrm{ml} 0.075 \mathrm{M} \mathrm{KCl}$ was added. Following this, the samples were incubated for $20 \mathrm{~min}$ at $37^{\circ} \mathrm{C}$, followed by centrifugation at $200 \mathrm{x} \mathrm{g}$ for $10 \mathrm{~min}$ at room temperature. The supernatant was discarded and cells were resuspended in $1 \mathrm{ml}$ cold fixative (methanol: Acetic acid, $3: 1)$, incubated for $0.5 \mathrm{~h}$ at room temperature, and washed three times at $200 \mathrm{x}$ g for $5 \mathrm{~min}$ each at room temperature. Finally, cells were suspended in 3 drops of the fixative. Slides were prepared by dropping a suspension of the pellet onto clean glass slides and dried at $37^{\circ} \mathrm{C}$ for $10 \mathrm{~min}$. Giemsa staining was performed for $30 \mathrm{~min}$ at room temperature, then it was examined under a light microscope (x100 magnification), followed by G-banding for analysis. Primarily, 20-30 good-quality (The mitotic phase was complete and independent and the chromosome of intact cells was observed) metaphases were screened. Chromosome abnormalities were described according to the standard of The International System for Human Cytogenetic Nomenclature (10).

Detection of the Janus kinase (JAK2)V617F mutation. Total DNA was isolated from bone marrow MNCs using the QIAamp DNA Blood Mini kit (Qiagen GmbH, Hilden, Germany), according to the manufacturer's protocols. DNA quality was assessed using a Bio-Rad Experion electrophoretogram instrument (Bio-Rad Laboratories, Inc., Hercules, CA, USA). The purity of the DNA was determined by measuring the optical density of the 260/280 ratio using a NanoDrop spectrophotometer. DNA samples were then stored at $-20^{\circ} \mathrm{C}$ for further use. All DNA samples were amplified in the C1000 Touch ${ }^{\mathrm{TM}}$ Thermal Cycler (Bio-Rad Laboratories, Inc.) using the Takara Premix Taq ${ }^{\mathrm{TM}}$ HS polymerase chain reaction (PCR) kit, UNG plus (Takara Bio, Inc., Otsu, Japan). In this system, a 50- $\mu 1$ reaction mixture contained the following components: $0.25 \mu \mathrm{l}$ Takara TaqHS DNA polymerase, $5 \mu \mathrm{l}$ 10X PCR buffer for UNG plus, $4 \mu \mathrm{l}$ dU plus dNTP mixture, $0.5 \mu 1 \mathrm{UNG}, 1 \mu 1$ each $20 \mathrm{pmol} / \mu \mathrm{l}$ primer, $2 \mu \mathrm{l}$ genomic DNA (200 ng) and $34.25 \mu \mathrm{l}$ diethyl pyrocarbonate-treated water. The mixture was amplified at $94^{\circ} \mathrm{C}$ for $4 \mathrm{~min}$ and subsequently subjected to 36 cycles of $94^{\circ} \mathrm{C}$ for $45 \mathrm{sec}, 60^{\circ} \mathrm{C}$ for $45 \mathrm{sec}$ and $72^{\circ} \mathrm{C}$ for $45 \mathrm{sec}$, and then extended at $72^{\circ} \mathrm{C}$ for $8 \mathrm{~min}$. Patients and controls were genotyped by a DNA tetra-primer Amplification Refractory Mutation Screening (ARMS) assay (11), a method that uses 
2 primer pairs to amplify the normal and mutant sequences plus a positive control band specifically in a single reaction. Primers included mismatches to maximize discrimination of the two alleles (in lowercase) and mutant/wild-type-specific bases (underlined). The PCR primers were as follows: Forward outer (FO), 5'-TCCTCAGAACGTTGATGGCAG-3'; reverse outer (RO), 5'-ATTGCT TTCCTT TTTCACAAGAT-3'; forward wild-type-specific (Fwt), 5'-GCATTTGGTTTTAAA TTATGGAGTATaT్-3', and reverse-mutant-specific (Rmt), 5'-GTTTTACTTACTCTCGTCTCCACAaAA-3' (The subsequent amplicons were electrophoresed on a $1.5 \%$ agarose gel and visualized following staining with ethidium bromide. The samples were visualized with a gel imaging system (Tanon 2500R; Tanon Science \& Technology Co., Ltd. Shanghai, China)

\section{Results}

Clinical characteristics and survival time. Among all the patients with AMKL, the white blood cell (WBC) count of 3 patients was normal, while 4 patients had a low WBC count and 2 patients had an increased WBC count. A total of 8 patients exhibited different degrees of anemia, 6 patients exhibited elevated lactate dehydrogenase levels, 6 patients exhibited hyperpyrexia and 6 patients exhibited bleeding from the skin or the mucosa. Peripheral blasts were observed in 6 patients, with a range of $2-30 \%$ (Table I).

The group consisted of 3 patients who exhibited marked splenomegaly. Additionally, patient no. 4 underwent splenectomy at diagnosis. A total of 2 patients exhibited superficial lymphadenopathy, 1 patient exhibited hepatomegaly (patient no. 6 underwent splenectomy due to trauma at diagnosis) and 1 patient exhibited sternal tenderness (Table I).

All patients were treated with cytarabine and daunorubicin/idarubicin. A total of 4 patients (patient nos. 3-6) achieved complete remission, 1 patient (patient no. 7) achieved partial remission and the remaining 4 patients exhibited no response. The median overall survival time of all patients was 6 months (range, 1.1-24.0 months).

Bone marrow smear and bone marrow cellularity. On the bone marrow smears, irregular forms of megakaryoblasts, pseudopodia or flocculence were frequently observed around the edge of the cytoplasm, which was occasionally accompanied by platelet aggregation (Fig. 1). Patient nos. 3 and 6 exhibited a notable increase in megakaryoblasts, as demonstrated by bone marrow aspiration, and the percentage of megakaryoblasts was 24 and $25 \%$, respectively. For the other patients, it was not possible to accurately identify the cells as megakaryoblasts by observing cell morphology, therefore, the percentage of megakaryoblasts was reported as $0 \%$. The bone marrow cellularity of these 2 patients (patient nos. 3 and 6) was moderately hypocellular or hypercellular, respectively (Table II). The percentage of bone marrow blasts in 2 patients (patient nos. 4 and 9) was $<5 \%$, while it was $>30 \%$ in 4 patients and it ranged from $20-30 \%$ in 3 patients (Table II).

Bone marrow biopsy and immunohistochemical staining. The bone marrow cellularity was also evaluated with a bone marrow biopsy. A total of 6 patients exhibited moderate or severe hypocellular bone marrow cellularity. The bone marrow cellularity of patient nos. 1, 2, 6 and 8 was severely, moderately, severely and moderately hypocellular, respectively, as demonstrated by bone marrow biopsy (Table III); however, the cellularity was hypercellular or moderate hypercellular, as indicated by bone marrow aspiration (Table II). The cellularity of the other patients was consistent between the results of the bone marrow aspiration and bone marrow biopsy. Gomori silver staining demonstrated that 7 patients had moderate or marked MF, and the remaining 2 patients were negative for the condition. Additionally, 7 patients exhibited the expression of platelet-specific antigens, including factor VIII, CD61, CD41 or CD42, as demonstrated by immunohistochemical staining (Fig. 2; Table III).

Immunophenotype. Immunophenotypic analysis was performed on all patients. The blast cells of 6 patients primarily expressed myeloid-specific antigens, including CD13 and CD33. A total of 3 patients primarily expressed platelet-specific antigens, including CD41 and CD61. The blast cell percentages of patient no. 4 and patient no. 9 were $<20 \%$ by bone marrow smear analysis; however, the percentage of CD41- and CD61-positive cells was $>90 \%$, as demonstrated by flow cytometry. In 5 patients, the percentage of CD34-positive cells was $>20 \%$ (Table IV).

Karyotypic analysis. A total of 6 patients exhibited an abnormal karyotype. Additionally, in 5 patients ( $5 / 6$ patients) complex karyotypes were observed. The karyotypes of patient nos. 3 and 5 contained the translocation $\mathrm{t}(9 ; 22)(\mathrm{q} 34 ; 11.2)$ (Table V).

JAK2V617F mutation. Primers FO and RO flanked the JAK2 exon 12 and generated a control 463-bp band in all patients. Primers Fwt and RO produced a 229-bp wild-type-specific product, and primers FO and Rmt generated a 279-bp mutant-specific product. Patient no. 6 was positive for the JAK2V617F mutation (Fig. 3).

\section{Discussion}

AMKL is a rare subtype of AML comprising only $~ 1 \%$ of all AML cases $(12,13)$. In China, a limited number of patients had been diagnosed with AMKL due to its low incidence. In the present study, only $1.4 \%$ of patients with AML were diagnosed with AMKL. In the clinic, AMKL may not be considered due to its low incidence. When clinicians diagnose AMKL, bone the marrow biopsy frequently exhibits proliferation of abnormal megakaryoblasts and extensive fibrosis (3). Immunohistochemical staining and flow cytometry frequently express platelet-specific antigens, including factor VIII, CD41, CD42 or CD61; however, no specific cytogenetic abnormalities are detected in patients with AMKL, including abnormalities of chromosomes 3 and 8 , and translocations, such as $\mathrm{t}(8 ; 17)$, $\mathrm{t}(1 ; 5)$ and $\mathrm{t}(10 ; 17)$, which have previously been reported in adult patients with AML-M7 (14-17).

In the clinic, patients may be diagnosed with AMKL upon finding $>20 \%$ of blasts and $>50 \%$ megakaryoblasts among all blasts, as determined by bone marrow aspiration; however, the diagnosis of AMKL is frequently challenging due to a high 


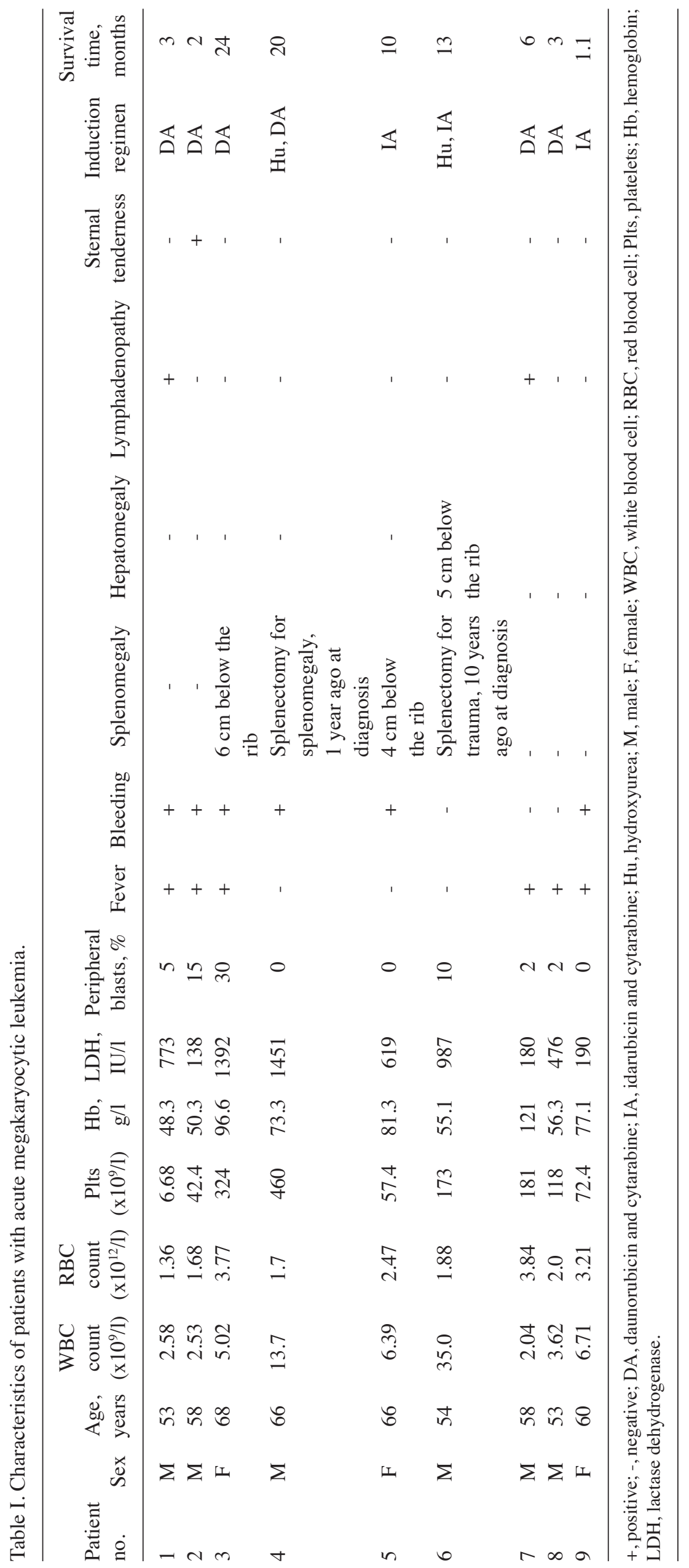


A

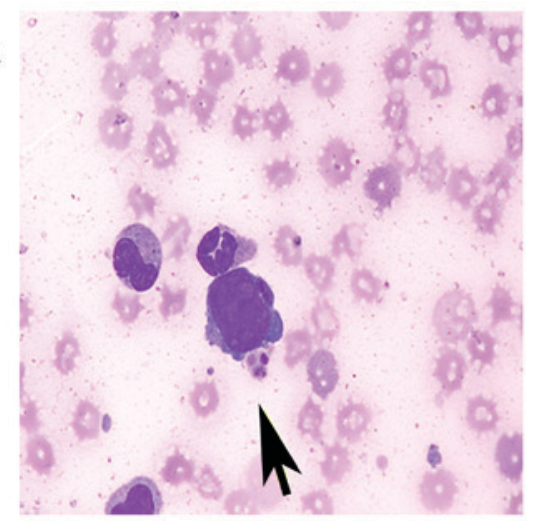

$\mathrm{B}$

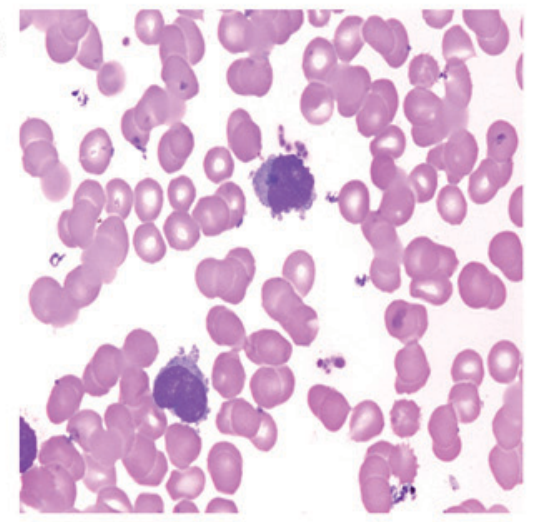

$\mathrm{C}$

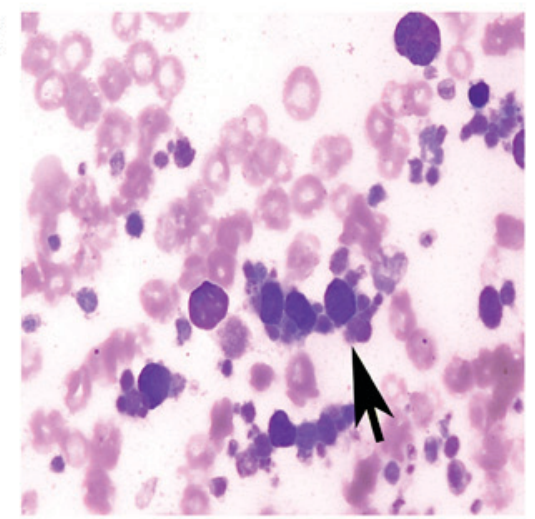

$\mathrm{D}$

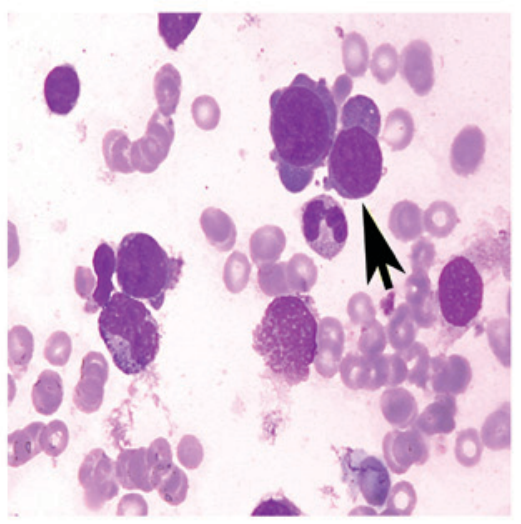

Figure 1. Cell morphology observation of bone marrow smears. Megakaryoblasts often displayed different cell morphology in patients with AMKL (A) Megakaryoblasts frequently present with a cytoplasmic budding and groups of aggregated platelets, which also present with (B) marginal flocculence and (C) production of large platelets. The amount of cytoplasm is variable, frequently with (C) a pale blue coloration or (D) mauve staining pattern of the granules. The nucleus is round, oval or irregular in shape, and fine-granular heterochromatin is also observed.
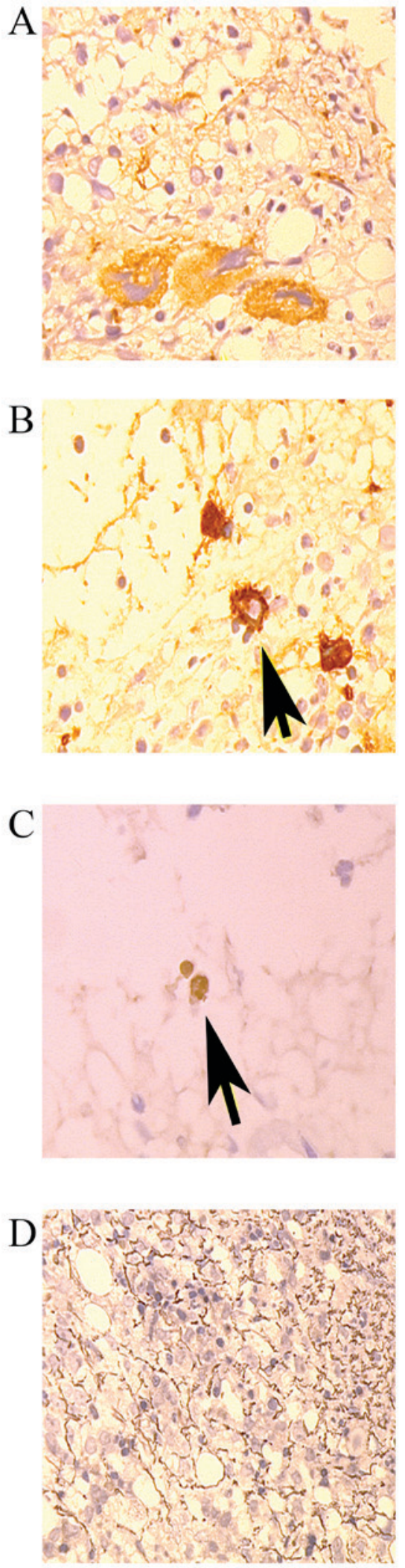

Figure 2. Immunohistochemical staining of bone marrow biopsy. (A) Immunocytochemical staining for factor VIII (magnification, x60) demonstrates that the expression of the marker is frequently positive. (B) The cell indicated by the arrow displays distinct blebs of the cytoplasm or pseudopod formation, and glycoprotein IIb/IIIa (CD41) (magnification) is positive. (C) The two cells indicated by the arrow are large cells present with irregular shapes, the small cell has a high nuclear-cytoplasmic ratio resembling lymphoblasts. The glycoprotein IIIa (CD61) (magnification, $\mathrm{x} 100$ ) staining of these cells is also positive. (D) Gomori staining frequently reveals myelofibrosis in patients with AMKL (magnification, x60). AMKL, acute megakaryocytic leukemia; $\mathrm{CD}$, cluster of differentiation. 
Table II. Bone marrow smear and cytochemical staining.

\begin{tabular}{|c|c|c|c|c|c|c|c|c|c|}
\hline $\begin{array}{l}\text { Patient } \\
\text { no. }\end{array}$ & $\begin{array}{l}\text { Bone marrow } \\
\text { cellularity }\end{array}$ & $\begin{array}{l}\text { Marrow } \\
\text { blasts, \% }\end{array}$ & Megakaryoblasts, $\%$ & $\begin{array}{c}\text { No. of } \\
\text { megakaryocytes }\end{array}$ & POX & PAS & NAE & SBB & $\mathrm{NCE}$ \\
\hline 1 & Hypercellular & 30.0 & 0 & 12 & - & +++ & +++ & $\mathrm{N} / \mathrm{A}$ & N/A \\
\hline 2 & Hypercellular & 60.5 & 0 & 6 & - & +++ & $++\sim+++$ & - & - \\
\hline 3 & Moderately hypocellular & 28.0 & 24 & $>500$ & - & +++ & ++ & - & - \\
\hline 4 & Severely hypocellular & 2.0 & 0 & 38 & ++ & $\mathrm{N} / \mathrm{A}$ & N/A & ++ & ++ \\
\hline 5 & Hypercellular & 31.0 & 0 & 89 & - & - & +++ & $\mathrm{N} / \mathrm{A}$ & $\mathrm{N} / \mathrm{A}$ \\
\hline 6 & Hypercellular & 29.0 & 25 & $>300$ & N/A & N/A & N/A & $\mathrm{N} / \mathrm{A}$ & $\mathrm{N} / \mathrm{A}$ \\
\hline 7 & Hypercellular & 59.0 & 0 & 21 & - & +++ & ++ & - & - \\
\hline 8 & Moderately hypercellular & 22.0 & 0 & 14 & - & ++++ & ++ & - & - \\
\hline 9 & Hypercellular & 3.5 & 0 & 143 & $\mathrm{~N} / \mathrm{A}$ & N/A & N/A & $\mathrm{N} / \mathrm{A}$ & $\mathrm{N} / \mathrm{A}$ \\
\hline
\end{tabular}

N/A, not performed; POX, Peroxidase; PAS, Periodic Acid- Schiff; NAE, Alpha-Naphythol/Acetate Esterase; SBB, Sudan Black B; NCE, naphythol AS-D chloroacetate esterase.

Table III. Bone marrow biopsy and immunohistochemical stain.

\begin{tabular}{|c|c|c|c|}
\hline Patient no. & Bone marrow cellularity & $\mathrm{MF}$ & Immunohistochemical stain \\
\hline 1 & Severely hypocellular & 0 & $\mathrm{FVIII}^{+} / \mathrm{CD} 61^{+}$ \\
\hline 2 & Moderately hypocellular & 0 & $\mathrm{FVIII}^{+++} / \mathrm{CD} 42^{+} / \mathrm{CD} 61^{+}$ \\
\hline 3 & Moderately hypocellular & 2 & $\mathrm{FVIII}^{+} / \mathrm{CD} 61^{+}$ \\
\hline 4 & Severely hypocellular & 3 & N/A \\
\hline 5 & Hypercellular & 3 & $\mathrm{FVIII}^{+} / \mathrm{CD} 42^{+} / \mathrm{CD} 61^{+}$ \\
\hline 6 & Severely hypocellular & 2 & N/A \\
\hline 7 & Hypercellular & 3 & $\mathrm{FVIII}^{+} / \mathrm{CD} 42^{+} / \mathrm{CD} 61^{+}$ \\
\hline 8 & Moderately hypocellular & 2 & $\mathrm{FVIII}^{+} / \mathrm{CD} 41^{+} / \mathrm{CD} 42^{+} / \mathrm{CD} 61^{+}$ \\
\hline 9 & Hypercellular & 3 & $\mathrm{FVIII}^{+} / \mathrm{CD} 1^{+} / \mathrm{CD} 42^{-}$ \\
\hline
\end{tabular}

FVIII, factor VIII; N/A, not performed; MF, marrow fibrosis; CD, cluster of differentiation.

incidence of MF, resulting in the failure of the bone marrow aspiration and inconsistency between aspiration and biopsy. In the present study, the bone marrow cellularity of patient nos. 1, 2, 6 and 8 was hypocellular to different degrees upon bone marrow biopsy; however, the cellularity was hypercellular upon bone marrow aspiration. Primarily, in other types of AML, if the bone marrow aspiration demonstrates hypocellular bone marrow cellularity, the result of the biopsy is frequently hypercellular; however, in AMKL, due to extensive $\mathrm{MF}$, following bone marrow aspiration, the samples selected by biopsy are frequently insufficient, therefore the opposite result occurs.

In the present study, 7 patients presented with moderate or extensive MF, which frequently results in 'dry taps' and diagnosis inaccuracy in the morphological examination of bone marrow; thus, in these 7 patients with MF, it was difficult to obtain the exact number of blast cells. Therefore, $>20 \%$ blasts and $>50 \%$ megakaryoblasts among the blasts were not essential diagnostic criteria for AMKL, and it was necessary to conduct a comprehensive diagnosis that depended on the results of bone marrow biopsy, flow cytometry and immunohistochemical staining. Furthermore, the diagnosis could not be confirmed under conditions where the percentage of blast cells was $<20 \%$ (18). In the present study, the percentage of blast cells in patient no. 4 and patient no. 9 was $<5 \%$, and this outcome may be associated with severe MF, which is demonstrated with strong positive Gomori staining or dilution of bone marrow blood; however, flow cytometry demonstrated the percentage of blast cells was $>20 \%$, and the expression levels of CD41 or CD61 were significantly increased (both $>90 \%$ ). Immunohistochemical staining also demonstrated that the expression of factor VIII was notably positive in patient no. 9, therefore leading to a diagnosis of AMKL.

Due to the rare incidence of AMKL and cost-saving considerations, conventional antibody combinations for surface antigens in diagnosing AML do not include CD41 and CD61, which may result in a missed diagnosis of AMKL. The 9 patients in the present study were not tested for the antigens of CD41 and CD61 at first, which made the diagnosis challenging depending on the results of cell morphology only. Finally, only 4 patients (patient nos. 1, 4, 6 and 9) underwent detection of expression levels of CD41 and CD61 by flow cytometry, and these patients were diagnosed with AMKL. The remaining 5 patients did not have data available to determine the 
expression levels of CD41 and CD61 antigens as they were not requested by the clinicians. In these 5 cases, the final diagnosis of AMKL depended on bone marrow biopsy and immunohistochemical staining. We suggest that when immunophenotypic analysis fails to diagnose AMKL, bone marrow biopsies should be analyzed by immunocytochemical staining for platelet-specific antigens, including factor VIII, CD41, CD42 or CD61. Compared with other types of leukemia, flow cytometry, bone marrow biopsy and immunocytochemical staining produce more notable results when patients are diagnosed with AMKL; thus, a comprehensive diagnosis in AMKL should be emphasized. We recommend that CD41 and CD61 analysis should be contained in the diagnosis of AMKL, if available.

Furthermore, clinical presentations may indicate the cause of AMKL and assist clinicians with diagnosing this type of disease accurately. In AMKL, 20-30\% of patients frequently exhibit splenomegaly and hepatomegaly, without lymphadenopathy $(12,13)$. In the present 9 patients, 3 exhibited splenomegaly, 1 exhibited hepatomegaly, possibly due to compensatory hepatomegaly (this patient underwent splenectomy for trauma 10 years ago prior to diagnosis), and 2 patients exhibited superficial lymphadenopathy. Patient no. 4 underwent splenectomy 1 year ago prior to diagnosis for unexplained splenomegaly and intolerable symptoms, and the diagnosis has now been confirmed as AMKL. It was considered that this patient may have had a history of myeloproliferative neoplasm (MPN). The patient was diagnosed with AMKL; however they may have had a history of MPN, meaning that AMKL may have transformed from MPN, therefore, they were diagnosed with secondary AMKL.

Notably, chromosomal karyotypic analysis may also indicate the cause of AMKL. Patient nos. 3 and 5 exhibited translocation of chromosomes 9 and $22, \mathrm{t}(9 ; 22)$, which primarily occurs in chronic myeloid leukemia (CML) (19). In patients with AMKL, the $\mathrm{t}(9 ; 22)$ chromosomal translocation may be exhibited in the acute stages of CML (20). Patient nos. 3 and 5 had no prior history of CML, and it is possible that CML was not previously diagnosed; thus, the diagnosis may be the blast crisis of CML. In the clinic, primary AMKL with Philadelphia chromosome is rare; therefore, this type of disease is frequently considered as secondary AMKL (21). Secondary AMKL is frequently reported from the transformation of CML, essential thrombocytosis (ET) and primary MF (PV) $(20,22,23)$. In the present study, 2 patients exhibited the Philadelphia chromosome (patient nos. 3 and 5), and patient no. 4 had a history of splenomegaly, which may be associated with MPNs. Furthermore, it was considered that the proportion of secondary AMKL was notably high. Additionally, all patients also underwent the detection of the JAK2 gene mutation, for which patient no. 6 was positive. This patient underwent a splenectomy due to trauma 10 years ago at diagnosis, and whether or not the spleen was enlarged at the time was unclear. However, JAK2V617F is frequently exhibited in ET and PV $(11,24,25)$, but not in AMKL, according to at least one report (26). Thus, it was speculated that the transformed AMKL of this patient was secondary to MPN.

In previous reports, patients with AMKL have short survival times and a poor prognosis, with median survival times that range from 5-10 months $(12,13,15,27)$. In the 9 patients in the present study, the median survival time 
Table V. Karyotype of patients with acute megakaryocytic leukemia.

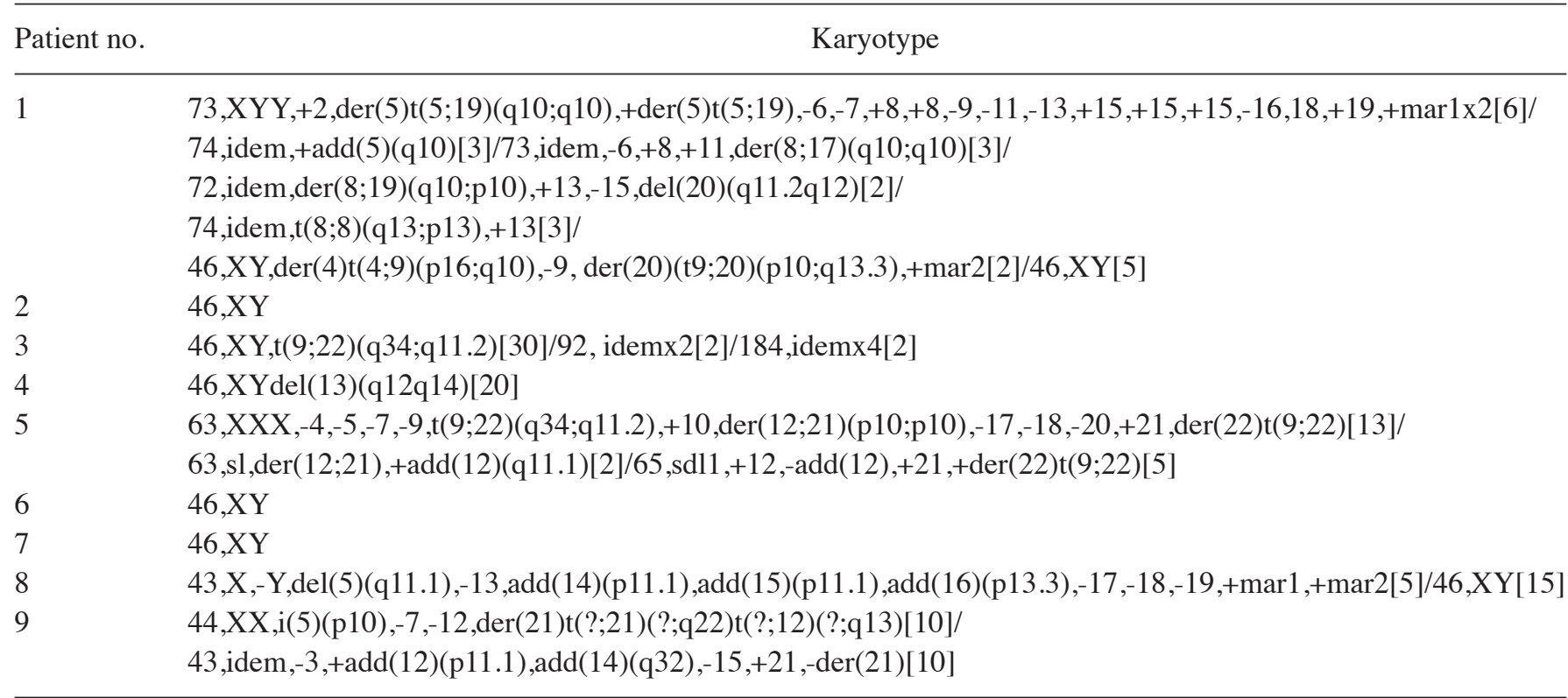

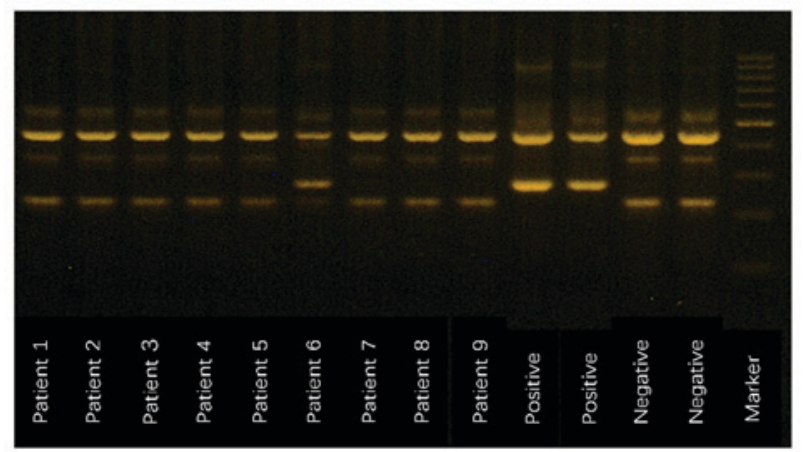

Figure 3. Amplification Refractory Mutation Screening assay to detect the JAK2V617F mutation in genomic DNA. Agarose electrophoresis analysis of the produced amplicons of the JAK2V617F gene mutation by reverse transcription-quantitative polymerase chain reaction. Patient no. 6 exhibited a mutan band, while other patients exhibited normal genotypes. JAK, Janus kinase.

was 6 months, which was consistent with the median survival time reported from other groups outside of mainland China $(12,13,15,27)$. Thus, it was considered that the survival time may be associated with the high proportion of secondary AMKL, bone marrow fibrosis and a complex karyotype. If patients with AMKL receive a regimen of cytarabine combined with anthracycline, $\sim 50 \%$ patients could achieve complete remission (15). Compared with traditional chemotherapy, allogeneic hematopoietic stem cell transplantation is a better choice in patients with complete remission, and benefits could be observed from this mode of therapy (28); however, the recurrence rate is high and the survival time is notably short (29). Novel treatment regimens require investigation for this type of disease in the future.

\section{Acknowledgements}

The authors would like to thank the Sino-U.S. Shanghai Leukemia Cooperative Group for their efforts.

\section{Funding}

The present study was supported by a grant awarded by the Key Construction Building Subject of a Three-Year Action Plan of the Fourth Round of a Public Health Project, Shanghai, China (grant no. 15GWZK0801).

\section{Availability of data and materials}

The datasets used and/or analyzed during the current study are available from the corresponding author on reasonable request.

\section{Authors' contributions}

JG and XW designed the experiments; GZ performed and analyzed most of the experiments; WW performed some experiments; GZ wrote the manuscript. All authors read and approved the final manuscript.

\section{Ethical approval and consent to participate}

All procedures performed in studies involving human participants were approved by the Ethics Committee of Huashan Hospital, Fudan University and in accordance with the 1964 Helsinki declaration and its later amendments or comparable ethical standards. Informed consent was obtained from all individual participants included in the study.

\section{Patient consent for publication}

Informed consent was obtained from all individual participant included in the study.

\section{Competing interests}

The authors declare that they have no competing interests. 


\section{References}

1. Von Boros $\mathrm{J}$ and Korenyi A: Uber einen fall von akuter megakaryocyblasten-leukamie, zugleich einige bemerkungen zum Problem der akuten leukemie. Z Klin Med 118: 679-718, 1931 (In German).

2. Breton-Gorius J, Reyes F, Duhamel G, Najman A and Gorin NC: Megakaryoblastic acute leukemia: Identification by the ultrastructural demonstration of platelet peroxidase. Blood 51: 45-60, 1978.

3. Bennett JM, Catovsky D, Daniel MT, Flandrin G, Galton DA, Gralnick HR and Sultan C: Criteria for the diagnosis of acute leukemia of megakaryocyte lineage (M7). A report of the French-American-British Cooperative Group. Ann Intern Med 103: 460-462, 1985

4. Swerdlow SH, Campo E, Harris NL, Jaffe ES, Pileri SA, Stein H, Thiele J and Vardiman JW: WHO Classification of Tumours of Haematopoietic and Lymphoid Tissues, 4th edition Lyon, pp136-137, 2008.

5. den Ottolander GJ, te Velde J, Brederoo P, Geraedts JP, Slee PH, Willemze R, Zwaan FE, Haak HL Muller HP and Bieger R: Megakaryoblastic leukaemia (acute myelofibrosis): A report of three cases. Br J Haematol 42: 9-20, 1979.

6. Strauchen JA: Criteria for the diagnosis of acute megakaryocytic leukemia. Blood 97: 1898, 2001.

7. Shibata A, Bennett JM, Castoldi GL, Catovsky D, Flandrin G, Jaffe ES, Katayama I, Nanba K, Schmalzl F, Yam LT, et al: Recommended methods for cytological procedures in haematology. International committee for standardization in haematology (ICSH). Clin Lab Haematol 7: 55-74, 1985.

8. Gruppo RA, Lampkin BC and Granger S: Bone marrow cellularity determination: Comparison of the biopsy, aspirate, and buffy coat. Blood 49: 29-31, 1977.

9. Thiele J, Kvasnicka HM, Facchetti F, Franco V, van der Walt J and Orazi A: European consensus on grading bone marrow fibrosis and assessment of cellularity. Haematologica 90: 1128-1132, 2005.

10. Gonzalez GJ and Meza-Espinoza JP: Use of the International System for Human Cytogenetic Nomenclature (ISCN). Blood 108: 3952-3953, 2006.

11. Jones AV, Kreil S, Zoi K, Waghorn K, Curtis C, Zhang L, Score J, Seear R, Chase AJ, Grand FH, et al: Widespread occurrence of the JAK2 V617F mutation in chronic myeloproliferative disorders. Blood 106: 2162-2168, 2005.

12. Pagano L, Pulsoni A, Vignetti M, Mele L, Fianchi L, Petti MC, Mirto S, Falcucci P, Fazi P, Broccia G, et al: Acute megakaryoblastic leukemia: Experience of GIMEMA trials. Leukemia 16: 1622-1626, 2002.

13. Tallman MS, Neuberg D, Bennett JM, Francois CJ, Paietta E, Wiernik PH, Dewald G, Cassileth PA, Oken MM and Rowe JM: Acute megakaryocytic leukemia: The Eastern Cooperative Oncology Group experience. Blood 96: 2405-2411, 2000.

14. Duchayne E, Fenneteau O, Pages MP, Sainty D, Arnoulet C, Dastugue N, Garand R and Flandrin G; GroupeFrançais d'Hématologie Cellulaire; Groupe Français de Cytogénétique Hématologique: Acute megakaryoblastic leukaemia: A national clinical and biological study of 53 adult and childhood cases by the Groupe Francais d'Hematologie Cellulaire (GFHC). Leuk Lymphoma 44: 49-58, 2003.

15. Oki Y, Kantarjian HM, Zhou X, Cortes J, Faderl S, Verstovsek S, O'Brien S, Koller C, Beran M, Bekele BN, et al: Adult acute megakaryocytic leukemia: An analysis of 37 patients treated at M.D. Anderson Cancer Center. Blood 107: 880-884, 2006.
16. Ahmad F, Dalvi R, Das BR and Mandava S: Novel t(8;17)(q23; $\mathrm{q} 24.2)$ and $\mathrm{t}(9 ; 22)(\mathrm{p} 24.1 ; \mathrm{q} 12.2)$ in acute megakaryoblastic leukemia AML-M7 subtype in an adult patient. Cancer Genet Cytogenet 193: 112-115, 2009.

17. Wang E and Stoecker M: 'Cannibalistic' phagocytosis in acute megakaryoblastic leukemia (AML M7) with $\mathrm{t}(10 ; 17)$ (p15; q22). Leuk Lymphoma 51: 1944-1947, 2010.

18. Hahn AW, Li B, Prouet P, Giri S, Pathak R and Martin MG: Acute megakaryocytic leukemia: What have we learned. Blood Rev 30: 49-53, 2016.

19. Druker BJ: Translation of the Philadelphia chromosome into therapy for CML. Blood 112: 4808-4817, 2008.

20. Karkuzhali P, Shanthi V and Usha T: A case of chronic myeloid leukaemia presenting as megakaryocytic blast crisis (AML M7). Ecancermedicalscience 7: 375, 2013.

21. Akahoshi M, Oshimi K, Mizoguchi H, Okada M, Enomoto Y and Watanabe Y: Myeloproliferative disorders terminating in acute megakaryoblastic leukemia with chromosome 3q26 abnormality. Cancer 60: 2654-2661, 1987.

22. Radaelli F, Mazza R, Curioni E, Ciani A, Pomati M and Maiolo AT: Acute megakaryocytic leukemia in essential thrombocythemia: An unusual evolution. Eur J Haematol 69: 108-111, 2002.

23. Mesa RA, Li CY, Kettering RP, Schroeder GS, Knudson RA and Tefferi A: Leukemic transformation in myelofibrosis with myeloid metaplasia: A single-institution experience with 91 cases. Blood 105: 973-977, 2005.

24. Vianelli N, Baravelli S and Gugliotta L: Acute megakaryoblastic transformation of essential thrombocythemia. Haematologica 81: 288-289, 1996.

25. Patiño-Sarcinelli F, Knecht H, Pechet L, Pihan G, Savas L and Snyder LM: Leukemia with megakaryocytic differentiation following essential thrombocythemia and myelofibrosis. Case report and review of the literature. Acta Haematol 95: 122-128, 1996.

26. Swaminathan S, Madkaikar M, Ghosh K, Vundinti BR, Kerketta L and Gupta M: Novel immunophenotypic and morphologic presentation in acute myeloid leukemia (AML) with JAK2 V617F mutation. Eur J Haematol 84: 180-182, 2010.

27. Giri S, Pathak R, Prouet P, Li B and Martin MG: Acute megakaryocytic leukemia is associated with worse outcomes than other types of acute myeloid leukemia. Blood 124: 3833-3834, 2014.

28. Garderet L, Labopin M, Gorin NC, Polge E, Baruchel A, Meloni G, Ortega J, Vossen J, Bunjes D, Leverger G, et al: Hematopoietic stem cell transplantation for de novo acute megakaryocytic leukemia in first complete remission: A retrospective study of the European Group for Blood and Marrow Transplantation (EBMT). Blood 105: 405-419, 2005.

29. Ishiyama $\mathrm{K}$, Yamaguchi $\mathrm{T}$, Eto $\mathrm{T}$, Ohashi $\mathrm{K}$, Uchida $\mathrm{N}$, Kanamori H, Fukuda T, Miyamura K, Inoue Y, Taguchi J, et al: Acute megakaryoblastic leukemia, unlike acute erythroid leukemia, predicts an unfavorable outcome after allogeneic HSCT. Leuk Res 47: 47-53, 2016.

This work is licensed under a Creative Commons Attribution-NonCommercial-NoDerivatives 4.0 International (CC BY-NC-ND 4.0) License. 